\title{
液固两相离心葲内固体颗粒对液相湍流调制的 测试方法及机理
}

史宝成 ${ }^{1^{*}}$, 魏进家 ${ }^{2 *}$, 邱伊婕 ${ }^{1}$, 沈秋婉 ${ }^{1}$, 张兴凯 $^{1}$, 张引弟 ${ }^{1}$

1. 长江大学石油工程学院, 武汉 430100 ;

2. 西安交通大学化学工程与技术学院, 西安 710049

*联系人, E-mail: shibaoch@126.con; jjwei@ mail.xjtu.edu.cn

2017-10-11 收稿, 2018-01-28 修回, 2018-01-31 接受, 2018-04-11 网络版发表 国家自然科学基金(51225601, 51606013)、湖北省教育厅计划项目(D20171305)和长江青年科技创新团队基金(2015cqt01)资助

摘要固液两相离心葲的性能和效率受控于厡内的两相流动规律, 理解葲内的两相流动规律和磨蚀机理, 对于 合理设计和延长厡的使用寿命具有重要意义. 以离心厡内的固液两相流为研究对象, 通过搭建无搅拌固液两相流 PIV测试平台, 消除高速搅拌对原内流场的影响, 改进颗粒-流体的两相循环方式, 提高了实验的测试精度; 通过 POD方法分析低固相浓度时原的性能改善的原因, 探寻固体颗粒对原内液相湍流的调制机理. 进一步补充和完善 固液两相流水动力学理论体系, 为指导固液两相流高效的工业应用提供理论基础.

关键词液固两相流动, PIV 测试, 流动特性, 湍流调制

渣浆葲和泥沙泵等输送固液两相混合物的水泵 是泵类中的耗能大户, 在工农业各部门中应用广泛. 过流部件的磨损及效率低下是固液两相流离心原在 实际使用过程中存在的两大问题 ${ }^{[1]}$, 不仅降低了固液 泵的可靠性, 缩短了使用寿命, 而且造成了设备和能 源的极大浪费. 目前, 现有的设计理论大都以单相流 一元或理想化单相流二元设计理论为基础, 而实际 运行中含有大量固体颗粒的两相流物理性质和特性 已经发生了改变. 长期以来, 由于设计理论和方法的 不完善导致设计条件与使用条件不相符, 从而造成 了两相洜的各性能指标恶化. 因此, 如何提高洜的效 率, 改善泵的性能, 一直都是国家和企业的重点研究 项目 ${ }^{[2]}$. 众多研究表明, 固液两相泵的性能和效率受 控于原内的两相流动规律, 因此搞清楚原内的两相 流动规律和磨蚀机理, 对于合理设计和延长泵的使 用寿命具有重要意义. 由于固液泵抽送介质的千差
万别, 两相流动与单相流相比要复杂得多, 一是描述 运动的变量几乎增加一倍, 二是在于各相的体积浓 度、分散相颗粒的大小、各相的物理性质(密度、黏 度等)及相间的相对速度都可以在很大范围内变化, 这些都可以引起流动性质和流动形态的变化. 因此, 不同类型的流动必须用不同的方法处理，而对于离 心原内的高旋转两相流场其运动更为复杂, 目前对 其内部的流动机理还无法完全解释清楚 ${ }^{[3]}$.

流场测试是反映真实流场最直接最可靠的方法, 目前所有的数值模拟都需要流场测试来验证与实际 流场的符合程度. 两相流洜的测试主要包括泵内颗 粒平均速度分布和浓度分布以及颗粒在叶道内的运 动及速度分布的测量. 目前, 探针、热线等常用的流 场测量方法已不适用于两相流场测量, 而非接触式 测量的粒子图像测速 (particle image velocimetry, PIV) 方法已成为公认的速度场测试的最有效方法. 而传 
统实验平台在进行葲内流场PIV测试实验时, 由于试 验工况复杂, 尤其在含固体颗粒的多相流条件下进 行原的试验时, 现有实验装置必须在水槽内附加动 力源搅拌装置 ${ }^{[4]}$, 才能使固液混合均匀、完成实验, 当测试颗粒密度比输送介质密度大得多时, 附加动 力搅拌速度就要求很高, 而高的转速就会带人大量 的气泡, 使得实验无法完成甚至失败. 针对这个问 题, 在前期的研究中已经设计出一套无搅拌的固液 两相实验平台, 有效地解决了高速搅拌带人气泡的 问题, 申请并授权了发明专利 ${ }^{[10]}$. 该实验平台用于 当前的研究中获得了很好的效果.

其次, 国内外对固液两相葲内流场的研究主要 集中在颗粒运动规律的数值模拟与试验研究上; 而 对于固体颗粒的加人对叶轮内液相湍流的调制作用 以及对原磨损机制的改变和对泵性能的影响机制研 究较少. 对于叶轮流动损失机制的研究多集中于单 相泵, 如Kruyt和Westra ${ }^{[11]}$ 为了更清楚地比较流场性 能, 引人Rothalpy作为能量损失的定量评价, 认为叶 片工作面相对速度变化较大, 比较容易出现分离流 动. 要获得比较理想化的相对速度分布, 就要使相对 速度在工作面附近的变化减小. 叶轮内的损失主要 集中在尾流区. 尾流是叶轮内边界层发展、二次流发 展、流动的分离和分层效应等相互影响、相互促进形 成的, 哥氏力是离心泵叶轮内部尾流形成的主要因 素, 但试验证明, 流体黏性对尾流形成也有不可忽视 的作用 ${ }^{[12]}$. 尾流区越宽, 射流-尾流剪切层越薄, 速 度梯度越大, 叶轮及蜗壳或导叶内的损失越大, 泵效 率越低 ${ }^{[13,14]}$. 这些从单相流洜分析得到能量损失机 制不能简单地应用到固液两相泵中, 需要充分考虑 固体颗粒的加人对液相湍流的调制作用, 探寻颗粒 尺寸效应、浓度效应对洜性能的影响. 有不少实验发

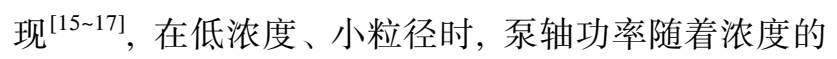
增加而增大; 泵的扬程和效率随着浓度的增加略有 升高. 这与张金风 ${ }^{[18]}$ 和许洪元 ${ }^{[19]}$ 分析得到的结论是 矛盾的, 他们的分析认为当百抽送密度比水大的固 体物料时, 在同样流量下, 其扬程小于抽送清水时的 扬程. 此外, 颗粒的加人对离心洜内大尺度涡的发展 演变机制研究很少, Quan等人 ${ }^{[20]}$ 从浮力效应的角度 分析螺旋泵内大尺度浴结构的演变过程. 浴旋的非 定常和强非线性使得涡旋的生成、扩散、溃灭、消失 的演变过程变得非常复杂. 因此, 本文在对㬌内固液 两相流动详细测试和分析的基础上, 采用特征正交 分解(proper orthogonal decomposition, POD)分析法, 探寻固体颗粒对洜叶轮内部液相湍流的调制机理以 及对洜性能的影响, 为两相泵的优化设计提供参考 依据.

\section{1 无摚拌固液离心原两相流PIV 实验测试 装置}

\section{1 实验装置和实验条件}

如图1所示, 本文设置一种新颖的固液两相流动 PIV测试系统, 包括电机、测试原、初始颗粒投放器 和储水罐; 电机与测试洜之间依次连接有转速调节 器、旋转轴编码器和转速转矩仪; 测试洜的出口管路 连接初始颗粒投放器的人口管路和两相循环管路; 两相循环管路的末端伸人储水罐中的固液两相泄能 器中; 固液两相泄能器的底部设有向下倾斜的出口 管路, 出口管路的末端为固液两相泄能器出口; 固液 两相泄能器出口伸人储水罐底部的储水罐出口管中, 储水罐出口管通过第一闸阀连接测试洜的人口管路; 初始颗粒投放器包括仿沙漏颗粒投放杯和雉形筒; 沙漏颗粒投放杯设置于雉形筒的顶部; 初始颗粒投 放器的人口管路伸人仿沙漏颗粒投放杯中，仿沙漏 颗粒投放杯的出口管路伸人雉形筒内; 仿沙漏颗粒 投放杯的出口管路上设有第二闸阀; 雉形筒的出口 连通测试泵的人口管路, 雉形筒的出口设有第三闸 阀. 测试洜选用透明材料制作, 拍摄时, 相机架设于 测试洜前与激光成垂直状态, 拍摄参数设置好后就 可以进行PIV实验测试了. 表1列出了试验台的主要 仪器 ${ }^{[21]}$. 在进行PIV测量时, 同步器同时与激光器、 编码器、高速相机和控制计算机连接, 并产生脉冲协 调高速相机和激光器的同步工作. PIV系统相关参数 被列在表2中.

本文设计的PIV试验台有以下特点: 循环管路出 口的多孔形式布置泄走了大部分的固液两相动能, 不至于因流速过高卷人大量气泡进入循环管路, 而 剩余的液体动能刚好可以通过颗粒两相循环泄能器 管路把固体颗粒带入到循环管路中, 颗粒两相循环 泄能器下端采用倒雉形的设计结构, 在实验设备运 行时, 颗粒两相循环泄能器内的液流产生足够的剪 切速度, 流体不同流层间由于速度差的存在相互混 合, 抑制了固体颗粒的自动沉降, 实现了固体颗粒与 输送介质的自动均匀混合. 此外, 颗粒投放装置仿沙 


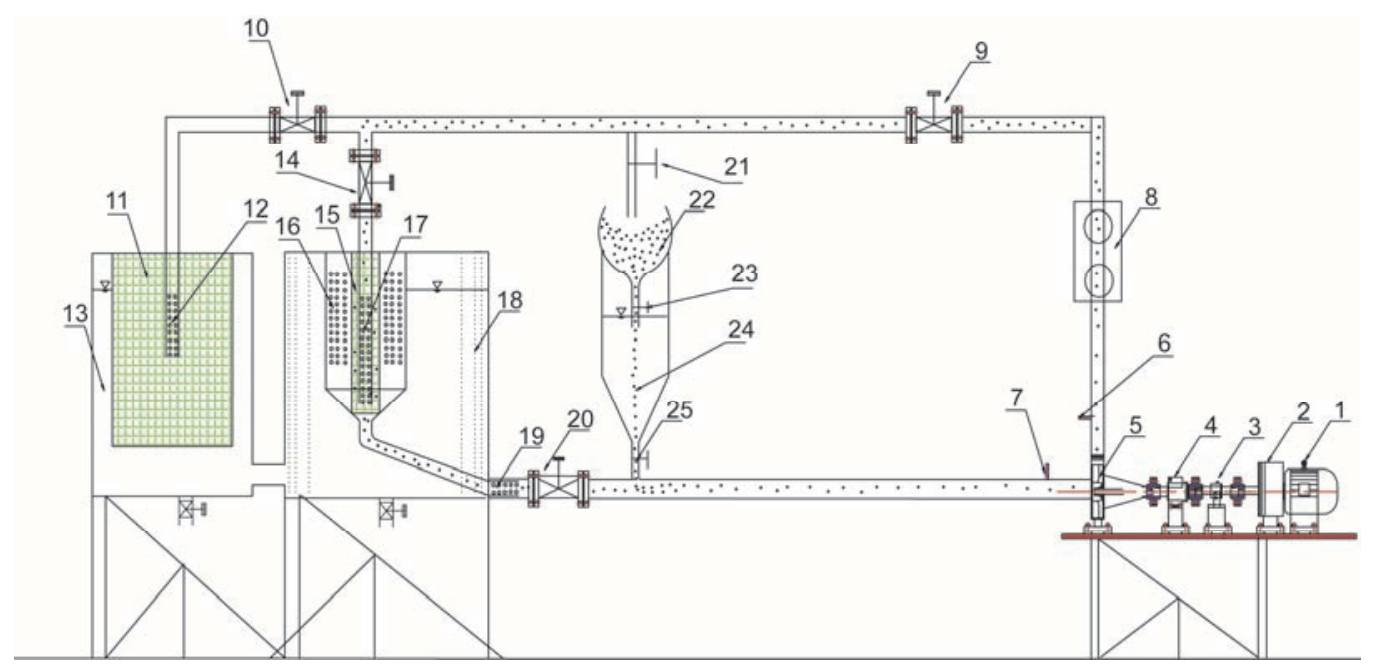

图 1 (网络版彩色) PIV 实验台二维示意图. 1, 电机; 2, 转速调节器; 3, 旋转轴编码器; 4, 转速转矩传感器; 5, 测试葲; 6, 7, 压力传感器; 8, 电 磁流量计; 9, 10, 14, 20, 21, 23, 25, 闸阀; 11, 滤网袋; 12, 固体颗粒回收管路出口; 13, 固体颗粒回收罐; 15 , 滤网圆筒; 16, 固液两相泄能器; 17, 两相循环管路出口; 18, 储水罐; 19 , 固液两相泄能器出口; 22, 仿沙漏颗粒投放杯; 24 , 初始颗粒投放器

Figure 1 (Color online) Schematic of test facility for PIV measurement of solid-liquid two-phase flow in centrifugal pump. 1, Motor; 2, stepless variable speed device; 3 , shaft encoder; 4 , torque and rotational speed measuring system; 5 , test pump; 6 , 7, differential pressure transducer; 8 , electromagnetic flowmeter; $9,10,14,20,21,23,25$, valve; 11, filter bag; 12, exports of solid particles recycle pipe; 13, solid particles recycle tank; 15, filter cylinder; 16, inverted cone perforated barrel; 17, perforated pipe; 18, water storage tank; 19, the porous outlet port; 22, the hourglass particle releasing cup; 24, particles releasing device

\section{表 1 试验台相关的主要仪器}

Table 1 The main devices of experiment appratus

\begin{tabular}{|c|c|c|c|}
\hline 序号 & 仪器名称 & 型号 & 精度 \\
\hline 1 & 无极变速器 & MBN & - \\
\hline 2 & 电磁流量计 & $\begin{array}{l}\text { KROHNE IFM } 4300 \mathrm{C} \text {, 内径 } D= \\
\quad 40 \mathrm{~mm}\end{array}$ & $\pm 0.5 \%$ \\
\hline 3 & 差压变送器 & LD3051J & - \\
\hline 4 & 轴编码器 & - & $1 / 1800$ \\
\hline 5 & 水箱和管道 & 316不锈钢 & - \\
\hline 6 & 转矩转速测量 & YP型和NJ & $\pm 0.5 \%$ \\
\hline 8 & 电机 & $\begin{array}{l}\text { 普通 } \mathrm{Y} \text { 系电机, 额定功率3 } \mathrm{kw} \text {, } \\
\text { 额定转速 } 1450 \mathrm{r} \mathrm{min}^{-1}\end{array}$ & - \\
\hline 9 & 液相/固相 & 水/玻璃球, 油菜籽 & - \\
\hline 10 & 试验温度 $\left({ }^{\circ} \mathrm{C}\right)$ & $\sim 25$ & - \\
\hline 11 & 曝光时间( $\mu \mathrm{s})$ & 100 & - \\
\hline 12 & PIV算法 & $\begin{array}{l}\text { DynamicStudio v3.41和变形窗口 } \\
\text { 算法 } \\
\end{array}$ & - \\
\hline
\end{tabular}

漏的设计结构可以保证颗粒均匀地释放到循环管路 中, 底部倒锥形的设计结构同样可以实现固体颗粒 与输送介质的自动均匀混合. 最后, 旋转编码器的加 人与PIV同步器和计数器的结合, 不仅可以保证泵内 流场PIV测试时每次拍摄的都是同一位置同一流道 的粒子图像, 而且可以设定获取任意角度叶片的粒 子图像. 本实验装置可用于单相介质状态下砅的外
特性、内流场等测试试验，也可用于固液两相流状态 下泵的外特性及内流场测试, 是一种多功能、适应多 种实验工况的离心泵PIV实验装置.

\section{2 实验内容}

本实验将针对普通叶片和复合型叶片两种叶轮, 采用PIV技术测量其内部流场, 并分析不同转速 $n$ 、不 同流量 $Q$ 和不同固体颗粒下模型泵的内部流动情况, 另外其他测量参数还包括泵前后压差、流量、泵转矩 转速等. 实验测试的工况见表 3 ; 用于试验测试的固 体颗粒实物图见图2.

图3给出了测量系统和测试洜的主要尺寸, 坐标 $X, Y, Z$ 分别对应葲的水平径向、泵流出口方向和洜流 人口方向, 方框 $\mathrm{A}$ 和 $\mathrm{B}$ 是拍摄窗口, $\mathrm{I}-\mathrm{I}$ 为拍摄断面. 在PIV测试过程中, 激光平行于壁面 $(X$ 方向), PIV相 机镜头平行于 $Z$ 方向. PIV系统购买自丹麦丹迪公司 (Dantec Dynamics A/S), 主要由 8 个部分组成, 激光、 高速相机、光源、同步器、时间触发器、电源、图像 处理软件和图像采集控制系统. 高速相机、同步器和 激光轴角编码器和控制计算机连接, 编码器生成脉 冲信号同步协调高速相机和激光工作. 示踪粒子尼 龙颗粒的大小为 $20 \mu \mathrm{m}$, 密度为 $1.05 \mathrm{~g} \mathrm{~cm}^{-3}$. 图 4 为 PIV测量系统现场图. 


\section{表 2 PIV测量系统参数}

Table 2 PIV system parameters

\begin{tabular}{|c|c|c|c|c|}
\hline 双腔 Nd: YAG激光器 & SpeedSense 9040相机 & 80N77同步控制器 & 示踪粒子 & 后处理软件 \\
\hline $\begin{array}{l}\text { 功率 } 2 \times 50 \mathrm{MJ} \text {; 波长 } 532 \mathrm{~nm} \text {; 脉冲宽 } \\
5 \sim 8 \mathrm{~ns} \text {; 频率 } 0 \sim 100 \mathrm{~Hz} \text {; 可调光栅 } \\
\text { 厚度 } 0.5 \sim 4 \mathrm{~mm} \text {; 角度 } 31^{\circ}\end{array}$ & $\begin{array}{l}\text { 帧率 } 1000 ; \text { 分辨率 } 1632 \times \\
1200 \text { pixel; EMS 内存 } 6 \\
\text { GB }\end{array}$ & $\begin{array}{l}\text { 通道数 } 8 \text {; 输人通道数 } 2 ; \text { 脉冲 } \\
\text { 定位精度 } 12.5 \mathrm{~ns} ; \text { 输出 TTL } \\
5 \mathrm{~V} \text { 和 } 50 \mathrm{Ohm} ; 80 \mathrm{MHz}\end{array}$ & $\begin{array}{c}\text { 尼龙, } \rho=1.05 \mathrm{~g} \mathrm{~cm}^{-3}, \\
d=20 \mu \mathrm{m}\end{array}$ & $\begin{array}{l}\text { Dynamic Stu- } \\
\text { dio } 3.4 .2\end{array}$ \\
\hline
\end{tabular}

\section{表 3 实验测试的工况情况 ${ }^{\text {a) }}$}

Table 3 The setting of testing working conditions ${ }^{\text {a) }}$

\begin{tabular}{lccccc}
\hline 固相颗粒 & 平均粒径 $d s(\mathrm{~mm})$ & 泵转速 $\left(\mathrm{r} \mathrm{min}^{-1}\right)$ & 流量 $\left(\mathrm{m}^{3} \mathrm{~h}^{-1}\right)$ & 固体颗粒浓度体积浓度 $(\%)$ & 拍摄断面 \\
\hline 油菜籽 & 2 & 900 & $0 \sim 1.2 Q_{0}$ & $1,5,10$ & $\mathrm{I}-\mathrm{I}$ \\
玻璃微珠 & $0.5,1.0$ & 900 & $0 \sim 1.2 Q_{0}$ & $1,5,10$ & $\mathrm{I}-\mathrm{I}$ \\
\hline
\end{tabular}

a) 因为实验材料的限制和安全考虑, 实际测试转速比额定转速低
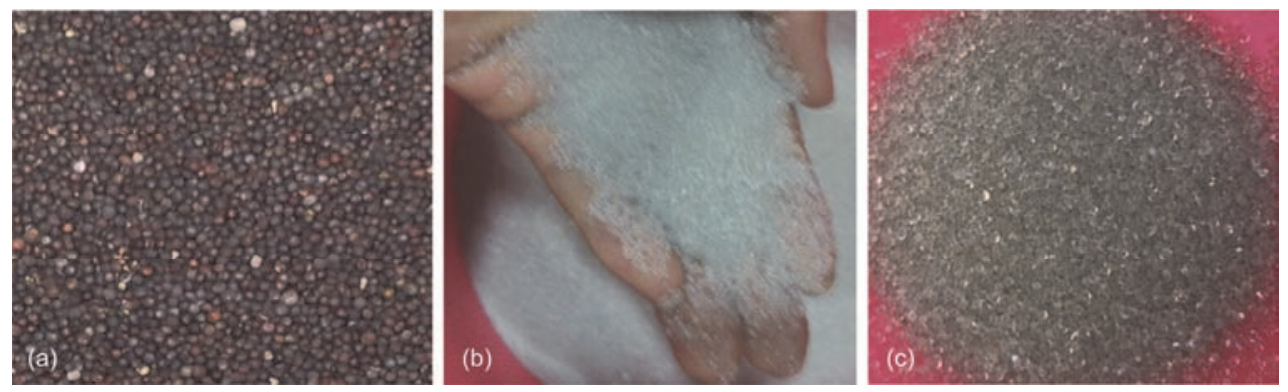

图 2 (网络版彩色) 3 种测试颗粒实物图. (a) 油菜籽 $(1.7 \sim 2.3 \mathrm{~mm}$ ); (b) 玻璃微珠 $(0.4 ~ 0.6 \mathrm{~mm})$; (c) 玻璃微珠 $(0.8 \sim 1.2 \mathrm{~mm})$

Figure 2 (Color online) The photos of three test-particles. (a) Rapeseeds, 1.7-2.3 mm; (b) glass beads, 0.4-0.6 mm; (c) glass beads, $0.8-1.2 \mathrm{~mm}$
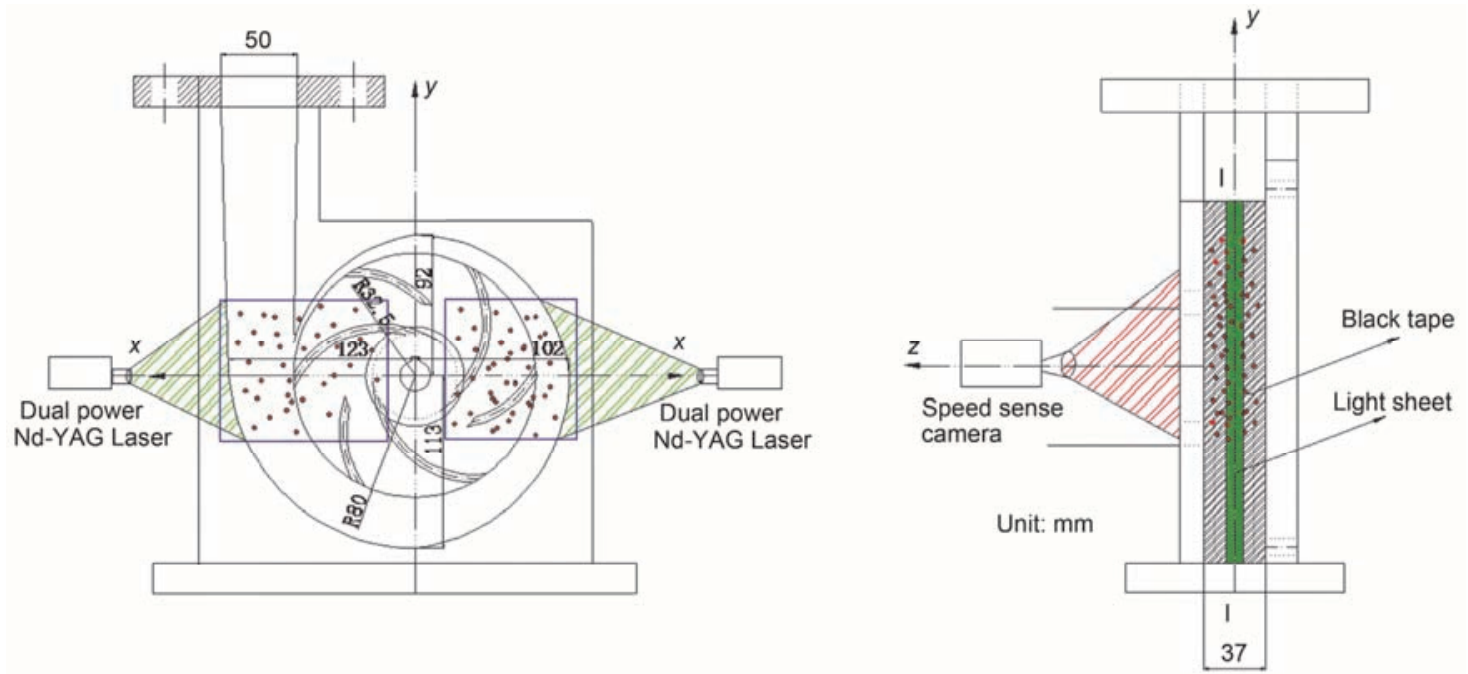

图 3 (网络版彩色)PIV测量系统和测试百主要尺寸 $(\mathrm{mm})$

Figure 3 (Color online) PIV measurement system and the main dimensions of test pump (mm)

\section{2 实验结果及分析}

\section{1 有无搅拌PIV测试平台的比较}

为了检验试验台获得数据的效果, 对比研究了 有搅拌和无搅拌两种情况下拍摄的粒子图片, 如图 5
所示. 可以发现，在有搅拌的情况下，当固体颗粒的 相对密度较大时需要高速搅拌来使固体颗粒悬浮, 从而带人大量气泡进人循环管路中, 因而所拍摄的 图片实际为气液固三相流(图5(a)), 而无搅拌的本文 设计的试验台就不存在这个问题, 得到的原始图片 


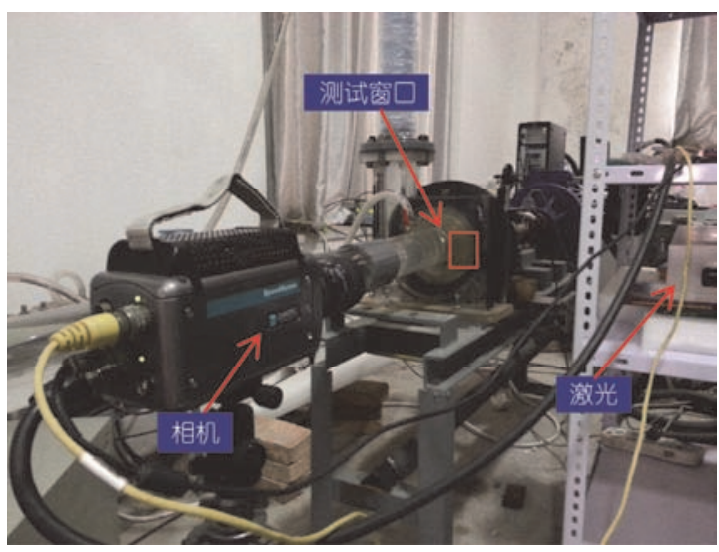

图 4 (网络版彩色)PIV测量系统现场图

Figure 4 (Color online) The photo of test facility for PIV measurement

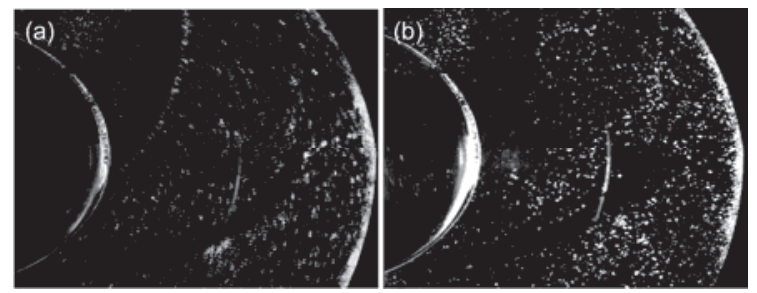

图 5 有无搅拌两种测试装置获得PIV粒子图像. (a) 有搅拌; (b) 无 搅拌

Figure 5 Particle image with and without agitating. (a) With agitating; (b) without agitating

非常清晰，没有卷人任何气泡(图5(b)).

\section{2 原叶轮内速度场测试结果}

PIV粒子图像采集方式采用粒子图像序列方式, 选用双脉冲模式, 每个测试点捕捉 50 帧共 25 对互相 关图像. 试验数据的处理过程分为两步: 先利用TSI 公司的dynamics 3.42软件, 运用互相关技术, 对每个 测试点的 25 对图像进行处理, 得到 25 个瞬时绝对速 度场, 再平均得到时均绝对速度, 并保存在相应的数 据文件中, 最后再采用Shi等人 ${ }^{[21]}$ 提出的高精度算法 进行精细处理. 离心原叶轮内流体的流动是一种复 合运动, 是由绕原轴转动的牵连运动、叶轮由里向外 流动的相对运动和相对于静止泵体的绝对运动组成 的. 根据绝对速度场、相对速度场和牵连速度场三者 的矢量关系, 通过编写程序对绝对速度、牵连速度进 行分解, 进而得到相对速度. 最后, 相对速度文件输 人绘图软件Tecplot中以实现矢量的显示.

(i ) 单相流洜叶轮内速度场分布特征. 图6所 示为泵转速 $n=900 \mathrm{r} \mathrm{min}$, 设计流量 $Q_{0}=15 \mathrm{~m}^{3} \mathrm{~h}^{-1}$. 由图6(a)和(b)可以看出, 在设计流量下压力侧绝对速 度较吸力侧高; 图 6(c) 和(d) 可以看出, 在设计流量 下, 在半径较小靠近叶轮进口时, 叶片吸力面附近相 对速度较高, 且最大值位于吸力面, 从吸力面到压力 面相对速度下降, 这与势流理论预测的流速分布一 致. 另外, 压力面附近的相对速度低, 与吸力面形成 较大相对速度差, 导致叶片表面负荷增大, 因而压力 面边界层有可能发生分离. 随着半径增大, 吸力面相 对流速增加缓慢, 而压力面增加较快, 两者的相对流 速差逐渐减小.

(ii) 固液两相流中叶轮内部夜相速度分布特点. 如图 7所示实验工况为 $Q=15 \mathrm{~m}^{3} \mathrm{~h}^{-1}$, 转速 $n=900 \mathrm{r}$ $\mathrm{min}^{-1}$. 图7给出了液相相对速度矢量分布, 可以看出 在液固两相流中, 由于固体颗粒的存在, 使得液体的 相对速度场发生了变化, 其出口速度要比单液相时 小, 图7中的吸力面和压力面的速度差比图6(d)中有 所降低, 在输送固体颗粒浓度 $C v>5 \%$ 时, 由于输送固 液两相流的混合密度大于单液相的密度, 在保持转 速不变的情况下, 输送两相流时离心原出口固相的 相对速度要比单相流时大, 这就导致了泵输送固液 两相流时扬程有所下降, 这与试验中测量的结果相 吻合. 在输送固体颗粒浓度较小时 $(C v<2 \%)$, 此时实 验测得的扬程比单相流时反而有所提高(图8), 而液相 相对速度出口分布与单相流时差别不明显, 这可能是 颗粒浓度较小时，颗粒浓度的增加抑制了小尺度涡的 发展，减少的脱流损失大于颗粒增加产生的摩擦损失.

图9分析了液相速度矢量和固相速度矢量沿径向 位置的变化, 可以看出在叶轮进口处固体颗粒和液 体的速度几乎相同. 出口处颗粒相的相对速度大于 液体的相对速度, 它们之间的速度差异在叶轮出口 处最大, 这可能是实验所用的固体颗粒直径较小 $(0.5 \mathrm{~mm})$, 与液体的跟随性较好. 未端出现两相最大 速度差, 意味着叶轮这部分容易出现冲击磨损. 本文 还进行了其他两种颗粒的实验 $(1.0 \mathrm{~mm}$ 的玻璃微珠和 $1.5 \mathrm{~mm}$ 的油菜籽), 其固液两相速度变化趋势与 0.5 $\mathrm{mm}$ 玻璃微珠相同, 差别主要在于 $1.0 \mathrm{~mm}$ 的玻璃微珠 在泵叶片出口处两相速度差异更明显, 而油菜籽的 固液两相速度差异不是那么明显, 可能是因为油菜 籽的密度与水相近, 跟随性较好.

\section{3 不同物理特性固体颗粒的运动特性分析}

试验中观察到在叶轮流道内, 固相体积浓度分 

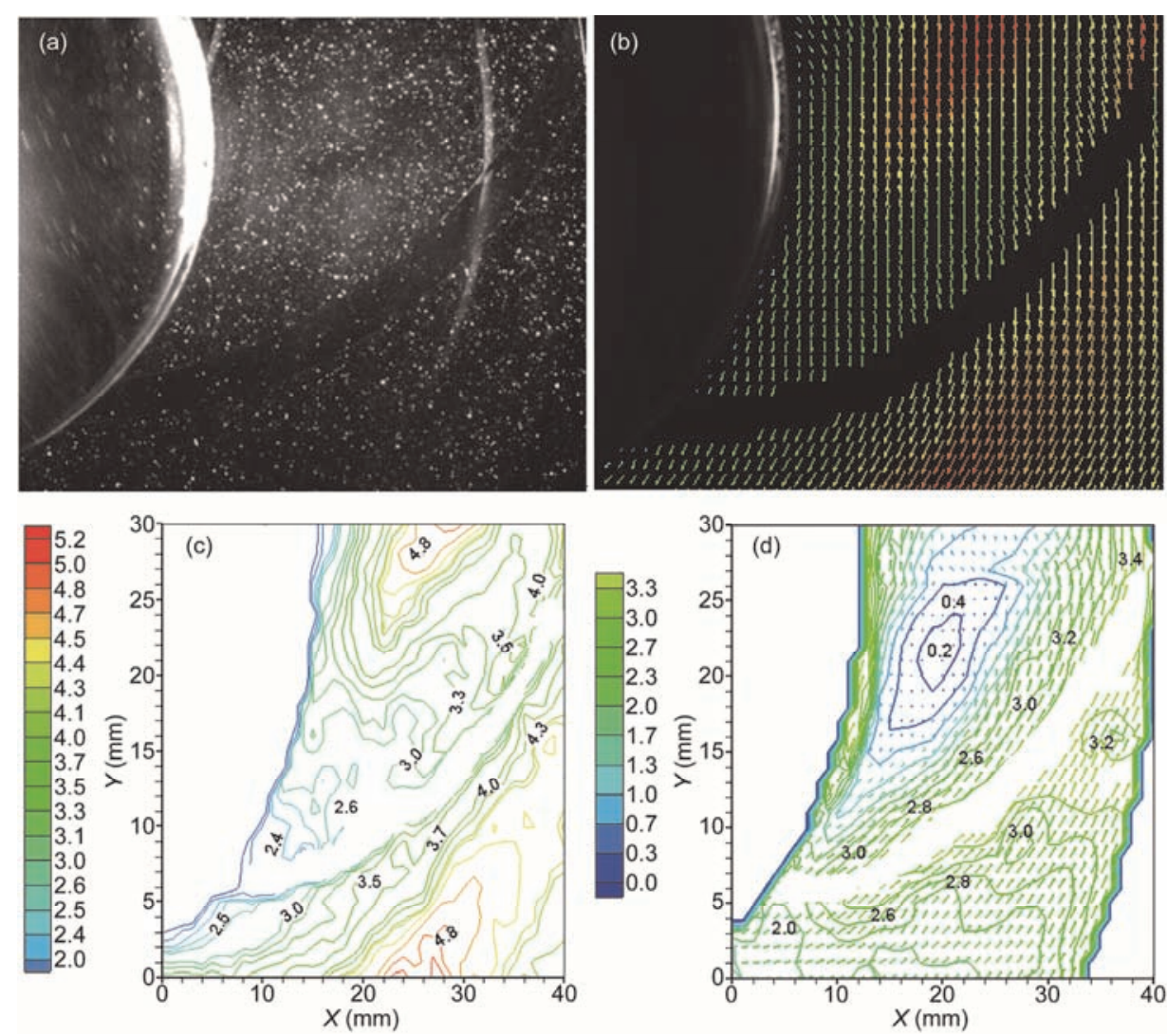

图 6 (网络版彩色)单相流时叶轮内的速度场分布 $\left(\mathrm{m} \mathrm{s}^{-1}\right.$ ). (a) 原始图像; (b) 绝对速度场分布; (c) 绝对速度分布; (d) 相对速度分布

Figure 6 (Color online) Velocity distribution in single-phase impeller flow $\left(\mathrm{m} \mathrm{s}^{-1}\right)$. (a) The original image; (b) the absolute velocity vector distribution; (c) the absolute velocity; (d) the relative velocity distribution

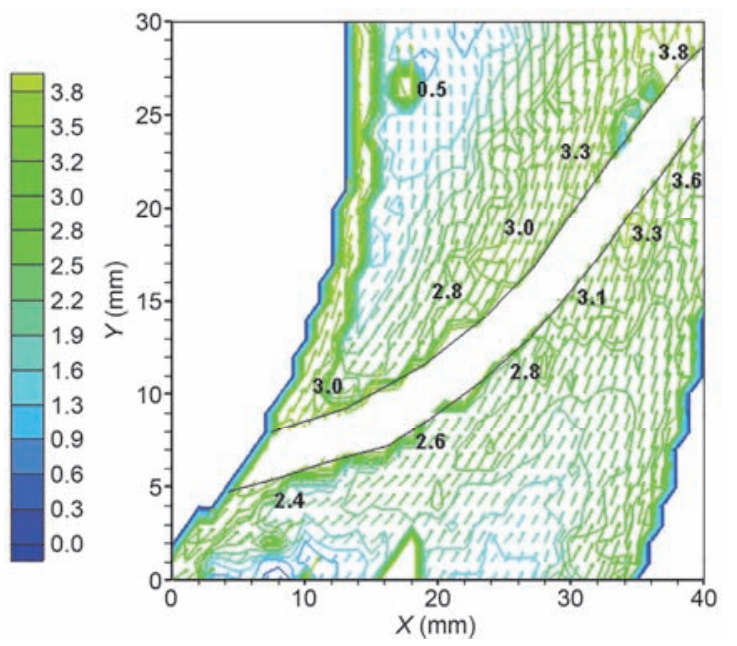

图 7 (网络版彩色)固液两相中相对速度分布(水-玻璃流, $d s=0.5$, $C v=5 \%)$

Figure 7 (Color online) The relative velocity distribution of solid-liquid two-phase flow ( water-glass flow, $d s=0.5, C \nu=5 \%$ )

布极不均匀, 在无叶腔内, 颗粒主要集中于靠近出口 侧的流道区域, 颗粒运动轨迹紊乱, 少部分颗粒脱离

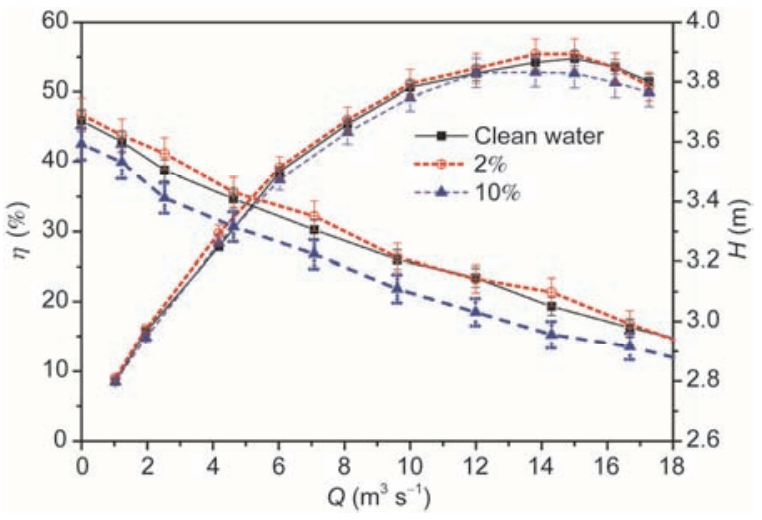

图 8 (网络版彩色)原性能曲线(玻璃微珠, $d s=0.5 \mathrm{~mm}$ )

Figure 8 (Color online) Pump performance curves (glass beans, $d s=$ $0.5 \mathrm{~mm}$ )

叶轮后能直接从出口流出，大部分颗粒撞击蜗壳壁 面, 留在无叶腔内转动数圈才能流出. 图10中可以看 出, 当输送固体颗粒密度较大时, 固体颗粒具有向压 力面移动的趋势, 这就导致了流道中压力面沉积玻 璃微珠较多. 实验中观察到的油菜籽、玻璃微珠等不 


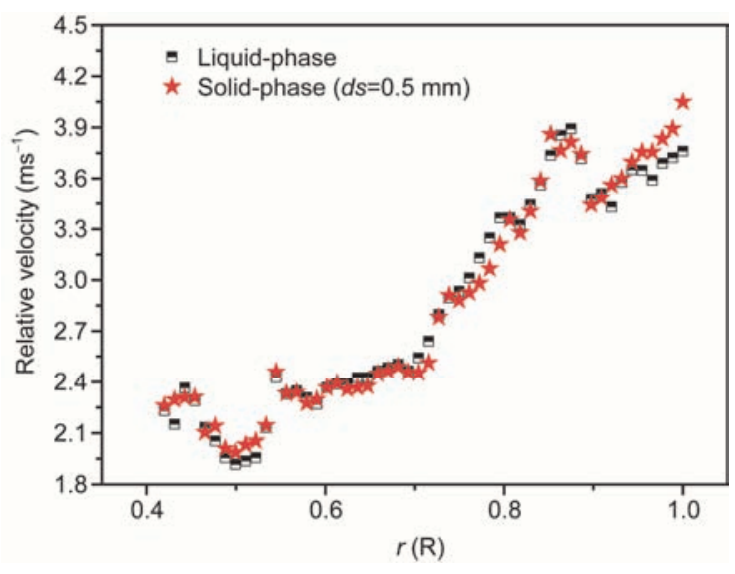

图 9 (网络版彩色)固液相沿着叶片压力面附近径向距离相对速度变 化 $\left(\mathrm{m} \mathrm{s}^{-1}\right)$

Figure 9 (Color online) The variation of solid-liquid relative velocity near pressure side with the radial range $\left(\mathrm{m} \mathrm{s}^{-1}\right)$

同物性的固体颗粒的运动特性见表4. 对于玻璃微珠, 随着粒径的增大, 离心力也越大, 会使颗粒集中于蜗 壳壁，颗粒越大，蜗壳壁磨损越快. 而对于密度较小 的油菜籽, 固体颗粒进入蜗室后不会很快移向蜗壳 壁, 颗粒在蜗室中的分布趋于均匀, 表明密度较小的 颗粒与水流的跟随比较好, 因而对蜗壳的冲蚀磨损 也较小.

\section{4 固体颗粒对液相湍流影响的POD分析}

POD方法的目的是把多维随机过程进行低维近 似描述并提取复杂随机过程的本质特征. 其基本思 想是将随机量分解为由其自身特征所确定的一组基 函数来表示, 基函数的确定原则为在每一次分解的 过程中使得最低阶的模式上含能最多. 它被广泛应 用于图像处理、信号处理和数据降维. POD数据分析 经常用来从高维实验数据或模拟数据中提取 “模式形 态”或基函数, 以此来产生低维的动态模数 ${ }^{[22]}$. POD 分析在此处有两个目的: (1) 离心原内的高速旋流是 典型的三维湍流, 相干结构的识别对于湍流的研究 非常重要. (2) 通过POD分析间接地验证本实验过程 泵内流动的稳定性和实验的可靠性.

(i ) POD快照法. POD分析方法包含连续POD, POD快照和时空对称POD 3种分析方法. 其中POD快 照方法简单易于实施, 目前被广泛用于PIV数据的处 理. POD快照方法 ${ }^{[23]}$ 需要一系列的脉动速度场 $u_{\mathrm{n}}(x)$, 这些脉动速度场取自同一个模拟工况或同一个测量 工况下不同时刻的数据. 只要保证速度场之间线性
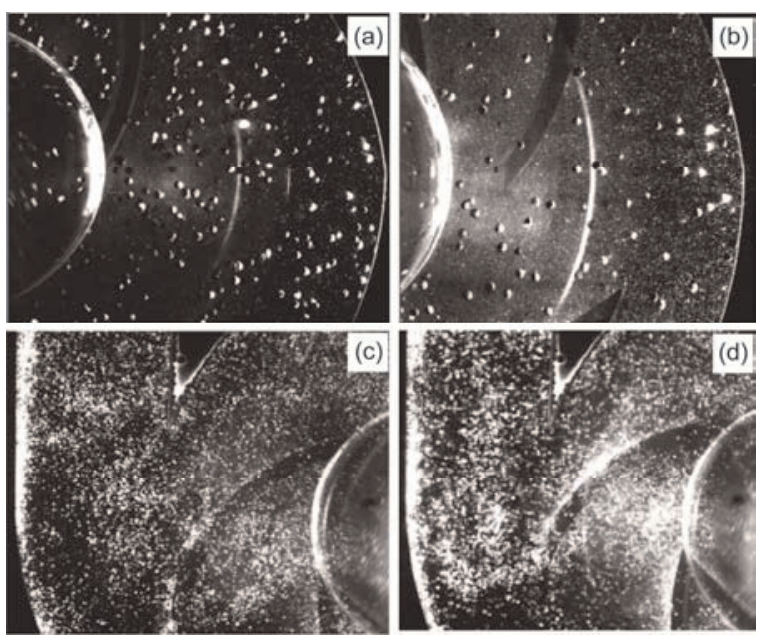

图 10 不同固体颗粒在叶轮中的分布情况. (a) 油菜籽颗粒分布(五 叶片, $C v=5 \%$ ); (b) 油菜籽颗粒分布(复合叶片, $C v=1 \%$ ); (c) 玻璃微珠 颗粒分布(五叶片, $d s=0.5, C v=8 \%$ ); (d) 玻璃微珠颗粒分布(五叶片, $d s=1.0, C v=8 \%)$

Figure10 Solid-particle distribution in impeller. (a) Rapeseeds (five blades, $C v=5 \%$ ); (b) rapeseeds (with splitter bladess, $C v=1 \%$ ); (c) glass beads (five blades, $d s=0.5, C v=8 \%$ ); (d) glass beads (five blades, $d s=1.0$, $C v=8 \%)$

无关即可, 而对时间间隔没有要求. 为了识别湍流相 干结构, Lumley ${ }^{[24]}$ 首次将POD引人到流体力学的研 究中. Lumley认为可以将相干结构看作为空间变量 $\boldsymbol{\Phi}(x)$ 的线性组合, 且 $\boldsymbol{\Phi}(x)$ 需在下式取得最 大值:

$$
H=\max _{\boldsymbol{\Phi}} \sum_{k=1}^{m} \frac{[\boldsymbol{\Phi}(x), u(x, t)]^{2}}{\|\boldsymbol{\Phi}(x)\|^{2}}, \boldsymbol{\Phi}(x)^{H} \boldsymbol{\Phi}(x)=\boldsymbol{I},
$$

通过优化技术可得

$$
J(\boldsymbol{\Phi}(x))=\sum_{k=1}^{m}(u(x, t), \boldsymbol{\Phi}(x))^{2}-\lambda(\|\boldsymbol{\Phi}(x)\|-1),
$$

对上式两边的 $\boldsymbol{\Phi}$ 求偏导数, 并令其为 0 , 整理 可得

$$
\left(\boldsymbol{X} \boldsymbol{X}^{H}-\lambda \boldsymbol{I}\right) \boldsymbol{\Phi}(x)=0,
$$

其中, $\lambda$ 是特征值; $\boldsymbol{\Phi}$ 为 $\lambda$ 对应的特征向量. 将求取最优 子空间问题转化为式(3)的特征值问题, 因为由 POD 基(特征向量)张成的最优子空间, 称 $\boldsymbol{X} \boldsymbol{X}^{H}$ 为 $\mathrm{POD}$ 核, 其维数为 $n \times n . u(x, t)$ 代表 $x$ 方向和 $t$ 时刻的速度场. 如 果 $\boldsymbol{\Phi}(x)$ 能使式 (3) 取得最大值, 这意味着流场沿着 $\boldsymbol{\Phi}(x)$ 投影的平均能量大于沿其他任何方向投影的平 均能量.

对于POD快照方法，分析要基于相同实验条件 下在相同位置获得一系列的流场快照, 在PIV的流场 


\section{表 4 不同固体颗粒在叶轮和蜗壳中的运动特征}

Table 4 Movement characteristics of different solid particles

\begin{tabular}{|c|c|c|c|}
\hline 项目 & 油菜籽 & 玻璃微珠 & 玻璃微珠 \\
\hline 颗粒类型 & 近球形, $\rho=1100 \mathrm{~kg} \mathrm{~m}^{-3}$ & 球形, $\rho=2500 \mathrm{~kg} \mathrm{~m}^{-3}$ & 球形, $\rho=2500 \mathrm{~kg} \mathrm{~m}^{-3}$ \\
\hline 运行参数 & $n=900 \mathrm{r} \mathrm{min}^{-1}, Q=15 \mathrm{~m}^{3} \mathrm{~h}^{-1}$ & $d s=0.4 \sim 0.6 \mathrm{~mm} n=900 \mathrm{r} \mathrm{min}^{-1}, Q=15 \mathrm{~m}^{3} \mathrm{~h}^{-1}$ & $d s=0.8 \sim 1.2 \mathrm{~mm}, n=900 \mathrm{r} \mathrm{min}^{-1}, Q=15 \mathrm{~m}^{3} \mathrm{~h}^{-1}$ \\
\hline 运动特性 & $\begin{array}{l}\text { 在进口和出口附近, 颗粒 } \\
\text { 相对速度方向总体趋势 } \\
\text { 与叶片曲线方向基本一 } \\
\text { 致. 在蜗壳中, 颗粒流动 } \\
\text { 方向接近于圆周的切线 } \\
\text { 方向 }\end{array}$ & $\begin{array}{l}\text { 固粒进人叶片间流道时, 其运动方向由轴 } \\
\text { 向转为径向, 由于惯性作用, 颗粒大多 } \\
\text { 移向后盖板. 在叶片间流道中, 相对速 } \\
\text { 度方向与叶片曲线方向较接近, 出口角 } \\
\text { 比较小. 蜗室中颗粒的速度方向接近于 } \\
\text { 圆周的切向. 在隔舌附近, 有一部分混 } \\
\text { 合液流随着叶轮旋转, 重新流人蜗室 }\end{array}$ & $\begin{array}{l}\text { 固粒进人叶片间流道时, 其运动方向由轴向转为 } \\
\text { 径向时, 由于惯性较大, 颗粒移向后盖板的运 } \\
\text { 动趋势比小颗粒更为明显. 径向速度比较小, } \\
\text { 导致颗粒与叶片进口碰撞机率增大. 颗粒离开 } \\
\text { 叶轮后移向蜗壳壁, 而叶轮外缘附近的颗粒相 } \\
\text { 对较少, 部分颗粒与蜗壳壁碰撞后, 离开蜗壳 } \\
\text { 壁, 分布于蜗室中. 在隔舌处, 有部分颗粒回 } \\
\text { 流到蜗壳流道 }\end{array}$ \\
\hline 分析结论 & $\begin{array}{c}\text { 表明密度较小的颗粒与水 } \\
\text { 流的跟随比较好 }\end{array}$ & $\begin{array}{l}\text { 叶轮出口的混合流的圆周速度分量比较大, } \\
\text { 使颗粒易于靠近叶片出口部分, 将导致 } \\
\text { 叶轮磨损加剧. 同时由于出口径向速度 } \\
\text { 分量比较小, 固粒进入蜗室后不会很快 } \\
\text { 移向蜗壳壁, 蜗室中的分布趋于均匀 }\end{array}$ & $\begin{array}{l}\text { 颗粒与叶片碰撞后离开压力面, 但随后在惯性作 } \\
\text { 用下将又向工作面靠近; 颗粒离开叶轮时, 相 } \\
\text { 对圆周速度分量比小颗粒的小, 对叶片出口的 } \\
\text { 作用相应地减小. 由于离心力较大, 会使颗粒 } \\
\text { 集中于蜗壳壁, 颗粒越大, 蜗壳壁磨损越快 }\end{array}$ \\
\hline
\end{tabular}

测试中, 每个瞬态PIV测量被看作为流场的一个快 照. 首先计算出所有快照中的平均流场 (平均流场被 看作为 POD的第 0 个模态), 然后计算出所有快照的 脉动速度场. 剩余的分析是对速度脉动场进行的. 将 $n$ 个速度快照的速度脉动分量排列在一个矩阵 $\boldsymbol{X}$ 中, 这意味着每列的数据来自一个给定的速度快照.

基于矩阵 $\boldsymbol{X}$, 可以得到一个自相关矩阵 $\boldsymbol{A}$ :

$$
\boldsymbol{A}=\boldsymbol{X} \boldsymbol{X}^{H} \text {. }
$$

事实上, 求解式(1)最大值的问题能被转化为求 解式(3)特征值的问题.

将每个特征值对应的特征向量与矩阵 $\boldsymbol{X}$ 组合就 可以计算出特征函数, 将特征函数标准化就得到 POD的模态

$$
\varphi_{i}=\frac{\boldsymbol{A} \boldsymbol{\Phi}_{i}}{\left\|\boldsymbol{A} \boldsymbol{\Phi}_{i}\right\|}, i=1,2, \cdots, n-1,
$$

其中, $\varphi_{i}$ 是对应特征值 $\lambda_{i}$ 和第 $i$ 个特征向量的 POD模态.

POD不仅可以用来识别流动的特征结构(即湍流 相干结构), 而且可以重构低维流场, 即用最少量的 模态来描述主要的流动. 每个POD快照能被POD模 态矩阵放大, 可得到放大系数 (也被称为 $\mathrm{POD}$ 系数)

$$
a_{i}=\boldsymbol{\Psi}^{\mathrm{T}} u_{i}, i=1,2, \cdots, n-1,
$$

其中, $\Psi$ 为POD模态矩阵, POD的每个模态是它的一列.

$$
\boldsymbol{\Psi}=\left[\begin{array}{llll}
\varphi_{1} & \varphi_{2} & \cdots & \varphi_{n-1}
\end{array}\right],
$$

只要知道POD系数 $a_{i}$, 就可以对相应的速度矢量 场进行重构

$$
u_{i}=\boldsymbol{\Psi} a_{i} .
$$

POD系数代表了流场 $u(x, t)$ 在方向 $\boldsymbol{\Phi}_{i}$ 上能量的大 小. 那么流场在不同方向上总的能量就是POD系数 之和

$$
E=[u(x, t), u(x, t)]=\left[a_{i}, a_{i}\right]=\sum_{i=1}^{n} a_{i}^{2}=\sum_{i=1}^{n} \lambda_{i},
$$

式(9)中的 $E$ 表示流动平均湍动能的两倍. 这样第 $i$ 个 模态占有能量的比例可表示为

$$
E_{i}=\frac{\lambda_{i}}{E} .
$$

从第一个模态到第 $m$ 个模态对总能量的累积贡 献为

$$
E_{i}^{\prime}=\sum_{i=1}^{m} \lambda_{i} / \sum_{i=1}^{n} \lambda_{i}
$$

当 $m=n$ 时, 能量的累积贡献率为 1 .

(ii) POD结果分析. 数据处理中发现, 不同工 况下的POD结果是非常相似的, 因此下面仅给出设 计工况下，颗粒浓度 $C v=5 \%$ 、玻璃微珠 $d s=1.0 \mathrm{~mm}$ 的 分析结果. 图 11 展示了模态能量比例随模态数的变 化情况. 可以看出, 模态数越小, 占有能量的比例越 高, 第一模态占有湍动能的比例最高. 从能量的角度 来看, 最重要的模态是第一模态. 通常意味着, 第一 模态对应的湍流涡结构的尺度最大. 从图11也可以 看出, 与单相流相比, 固体颗粒的注人提高了液相小 模态数能量的占有比例, 而减小了液相高模态数的 能量占有比例. 这意味着, 颗粒的加人对大结构的湍 
流含能浴有很大的影响. 可以看出, 与单相流相比, 液相的能量贡献率在前几个模态比单相流要大, 这 意味着固体颗粒的加人增加了大结构含能浴的能量 占有比例, 而减小了小尺度随机湍流结构的能量占 有比例, 使流动变得更加各向异性. 也就是说, 固体 颗粒的加人改变了能量的分配关系, 减小了小尺度 随机湍流结构对能量的耗散, 这就解释为什么在低 浓度固相体积浓度时, 泵的扬程和效率有所提高, 虽 然颗粒的加人增加了摩擦损失却减少了脱流损失, 抑制了小尺度浴的发展, 但是随着颗粒浓度的增加, 增加的摩擦损失大于脱流损失的减少, 泵的扬程和 效率自然就下降了. 这个结果被实验所证实(图 8). 图8可以看出, 泵输送较低固相体积浓度 $(C v=2 \%)$ 水 泵扬程和效率高于它用于清洁水和高固相体积浓度 $(C v=10 \%)$. 图 12给出不同的固相体积浓度下叶轮内 的湍流强度分布. 可以看出, 低固相体积浓度 $(C v=$ $2 \%)$ 具有较弱的湍流强度. 这表明少量固体颗粒的加 人可以抑制小尺度涡的发展, 减少流动分离损失, 因 此水洜扬程和效率有所增加. POD分析表明, 无论是 单相流或液相(图11)能量分数的误差限都很小, 这间 接验证泵内的流动是稳定的, 也证实本文设计新PIV 测试平台可以实现稳定的固液两相流测量, 比传统 方法具有更好的可靠性.

\section{3 结论}

本文以离心原内的固液两相流为研究对象, 通 过搭建无搅拌固液两相流PIV测试平台, 消除高速摚 拌对洜内流场的影响, 改进颗粒-流体的两相循环方 式, 提高了实验的测试精度. 基于百内固液两相流动
的PIV测试，观测了不同工况下泵内固体颗粒的运动 规律, 并通过POD方法分析低固相浓度时泵的性能 改善的原因, 探寻固体颗粒对泵内液相湍流的调制 机理, 主要结论如下.

(1) 提出了一种颗粒-流体两相自动均匀混合的 新方法. 该方法利用新颖的多孔倒圆雉设计产生不 同流层差异剪切速度来完成混合, 一是避免了搅拌 对测试流场的影响, 二是倒圆雉内液体产生的旋流 抑制了颗粒的自由沉降和聚集, 有助于提高固液两 相流场的PIV测试精度. 该法解决现有PIV固液两相 流测试中高密度固体颗粒因高速搅拌卷人气泡降低 实验测试精度的问题. 该法用于结构复杂的离心泵 的固液两相流测试中, 捕捉到的PIV粒子图像清晰, 具有高精度高稳定性, 明显优于传统方法.

(2) 试验中观察到在叶轮流道内, 固相体积浓度

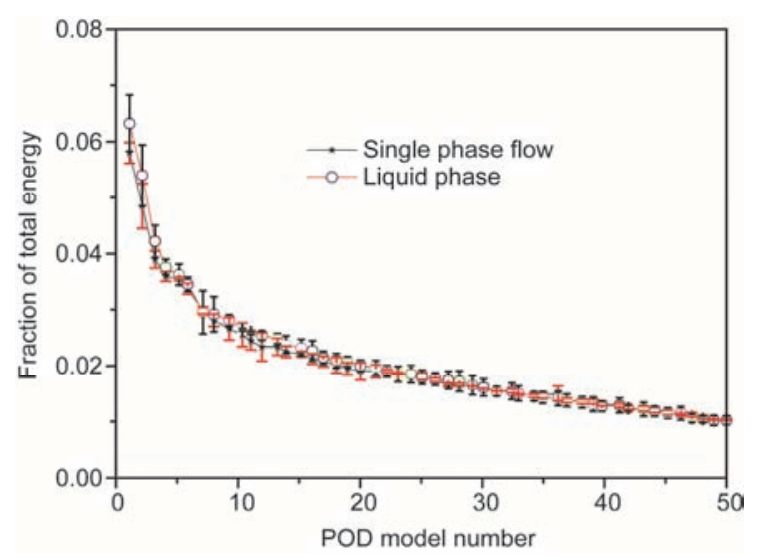

图 11 (网络版彩色)不同POD模态的能量分数

Figure 11 (Color online) Energy contribution of different POD mode number
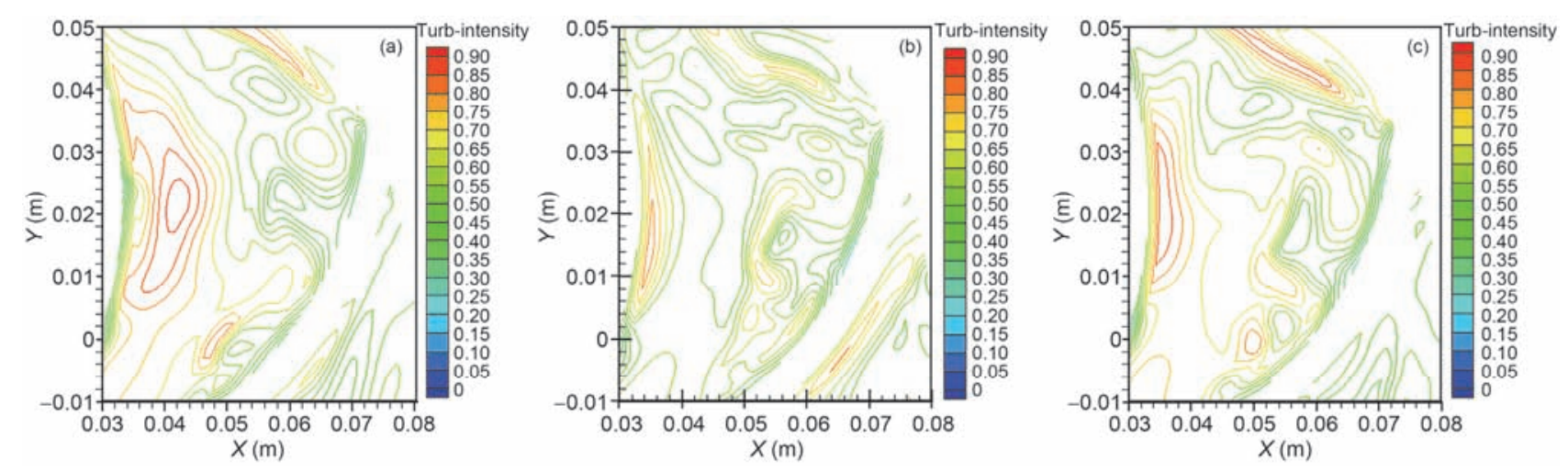

图 12 (网络版彩色)湍流强度分布. (a) 清水; (b) 玻璃微珠, $d s=0.5 \mathrm{~mm}, C v=2 \%$; (c) 玻璃微珠, $d s=0.5 \mathrm{~mm}, C v=10 \%$

Figure 12 (Color online) Turbulent intensities distribution. (a) Clean water; (b) glass beans, $d s=0.5 \mathrm{~mm}, C v=2 \%$; (c) glass beans, $d s=0.5 \mathrm{~mm}$, $C v=10 \%$ 
分布极不均匀，在无叶腔内，颗粒主要集中于靠近出 口侧的流道区域, 颗粒运动轨迹紊乱, 少部分颗粒脱 离叶轮后能直接从出口流出, 大部分颗粒撞击蜗壳 壁面, 留在无叶腔内转动数圈才能流出. 当输送固体 颗粒密度较大时, 固体颗粒具有向压力面移动的趋 势, 这就导致了流道中压力面沉积玻璃微珠较多.

(3) 基于PIV测量技术, 实验调查了固体颗粒加 人对液相湍流的影响. POD分析表明, 固体颗粒的注 人对含有大量能量的大尺度湍流结构有很大的影响.
固体颗粒的注人增加了大尺度湍流结构的能量占有 比例, 减小了代表能量耗散小尺度湍流结构能量的 占有比例. 也就是说，固体颗粒的加人改变了能量的 分配关系, 减小了小尺度随机湍流结构对能量的耗 散, 这就解释了为什么在低浓度固相体积浓度时, 洜 的扬程和效率有所提高, 虽然颗粒的加人增加了摩 擦损失却减少了脱流损失，抑制了小尺度浴的发展， 但是随着颗粒浓度的增加, 增加的摩擦损失大于脱 流损失的减少，泵的扬程和效率自然就下降了.

\section{参考文献}

1 Zhang J Z, Wang J, Yang J. Recent progress in solid-liquid two phase flow pump research (in Chinese). Energy Res Inform, 2014, 30: 1-6 [张敬斋, 汪军, 杨骏. 固液两相流洜的研究现状及展望. 能源研究与信息, 2014, 30: 1-6]

2 Zhao X B. Simulation and study of the flow field and the discrete solid particle trajectory in the slurry pump (in Chinese). Degree Dissertation. Beijing: Tsinghua University, 2002 [赵啸冰. 渣浆泵内清水流场及离散固体颗粒轨迹的模拟与研究. 博士学位论文. 北京: 清华大学, 2002]

3 Wang Y, Liu Y, Wang W J, et al. Numerical simulation of solid-liquid two phase flow in a double blades slurry pump (in Chinese). Chin Rural Water Hydropower, 2013, 11: 121-125 [王洋, 刘洋, 王维军, 等. 双叶片排污泵固液两相流的数值模拟. 中国农村水利水电, 2013, 11: 121-125]

4 Walker C, Wells P, Pomat C. The effect of impeller geometry on the performance of centrifugal slurry pumps. In: Proceedings of 4th Institution on Bulk Materials, Storage, Handing and Transportation. Melbourne: Institution of Engineers, 1992

5 Zhao J T. Study on Sand Pump, a Collection of Translated Essays in Two-Phase Flow Pump (in Chinese). Beijing: Tsinghua University Press, 1988 [赵敬亭. 砂泵的研究, 两相流泵译文集. 北京：清华大学出版社, 1988]

6 Dai J. Study on solid-liquid two phase flow in a centrifugal pump impeller (in Chinese). Degree Dissertation. Beijing: Tsinghua University, 1990 [戴江. 离心洜叶轮内固液两相流流动规律研究. 博士学位论文. 北京: 清华大学, 1990]

7 Xu H Y, Luo X W. Experimental research on effects of blades parameters on wear of centrifual pump(in Chinese). Trans CSAE, 1997, 4: 69-73 [许洪元, 罗先武. 离心原叶片参数对磨损规律影响的试验研究. 农业工程学报, 1997, 4: 69-73]

8 Wei J J. KET model and numerical calculation of dense phase liquid-solid two phase turbulence and experiment on two-phase flow in centrifugal pump impeller (in Chinese). Doctor Dissertation. Xi'An: Xi'An Jiaotong University, 1998 [魏进家. 密相液固两相湍流 KET 模型和数值计算及离心厡叶轮内两相流场实验. 博士学位论文. 西安: 西安交通大学, 1998]

9 Guo Z J, Zhao T C, Ren J, et al. Influence of impeller runner size on pump performance (in Chinese). Pump Tech, 1994, 5: 4-5 [郭自杰, 赵天成, 任俭, 等. 叶轮流道宽度对泵性能的影响. 水洜技术, 1994, 5: 4-5]

10 Wei J J, Shi B C, Zhang Y. Internal flow field PIV (Peak Inverse Voltage) testing device of stirring-free solid-liquid two-phase centrifugal pump. PRC Patent, CN 104500414 B, 2016-06-18

11 Kruyt N P, Westra R W. On the inverse problem of blade design for centrifugal pumps and fans. Inverse Probl, 2014, 30: 17-25

12 Wu B, Yan H Z, Duan Y Q. 3D numerical simulation on turbulentflow and wear characteristics in a slurry pump (in Chinese). Chin Mech Eng, 2009, 20: 719-722 [吴波, 严宏志, 段益群. 渣浆葲三维湍流数值模拟及磨损特性研究. 中国机械工程, 2009, 20: 719-722] Majidi K. Numerical study of unsteady flow in a centrifugal pump. J Turbomach, 2004, 127: 805-814

14 Barrio R, Parrondo J, Blanco E. Numerical analysis of the unsteady flow in the near-tongue region in a volute-type centrifugal pump for different operating points. Comp Fluid, 2010, 39: 859-870

15 Chen C C, Liu Z Y, Liu T B. Theory and Design of the Two-phase Flow Pump (in Chinese). Beijing: Weapon Industry Press, 1994. 36-41 [陈次昌, 刘正英, 刘天宝. 两相流原的理论与设计. 北京: 兵器工业出版社, 1994. 36-41]

16 Gopalakrishnan S. Pump research and development: Past, present, and future-an american perspective. J Fluid Eng, 1999, 121: 237-247

17 Gandhi B K G, Seshadri V. Performance characteristics of centrifugal slurry pumps. J Fluid Eng, 2001, 2: 271-280

18 Zhang J F. Numerical forecast and research on the design method for centrifugal pumps with splitter blades (in Chinese). Doctor Dissertation. Suzhou: Jiangsu University, 2007 [张金风. 带分流叶片离心百全流场数值预报和设计方法研究. 博士学位论文. 苏州: 江苏大学, 2007] 
19 Xu H Y. Theory study on solid-liquid two phase pump (in Chinese). J Coal Mach, 1997, 4: 19-21 [许洪元. 固液葲的两相流动理论研 究. 煤矿机械, 1997, 4: 19-21]

20 Quan H, Li R, Su Q, et al. Analysis on energy conversion of screw centrifugal pump in impeller domain based on profile lines. Adv Mech Eng, 2013, 2013: 512-523

21 Shi B C, Wei J J, Zhang Y. Phase discrimination and a high accuracy algorithm for PIV image processing of particle-fluid two-phase flow inside high-speed rotating centrifugal slurry pump. Flow Meas Instrum, 2015, 45: 93-104

22 Holmes P, Lumley J L, Berkooz G. Turbulence, Coherent Structures, Dynamical Systems and Symmetry, Cambridge Monogr Mecb. Cambridge: Cambridge University Press, 1996

23 Sirovich L. Turbulence and the dynamics of coherent structures. Part I: Coherent structures. Q Appl Math, 1987, 45: 561-571

24 Lumley J L. The structure of inhomogeneous turbulent flows. Atm Turb Radio Wave,1967, 166-178 


\title{
PIV test methods and mechanism on liquid turbulence modulation by solid-particles inside a centrifugal pump
}

\author{
Baocheng Shi ${ }^{1 *}$, Jinjia Wei ${ }^{2 *}$, Yijie Qiu ${ }^{1}$, Qiuwan Shen ${ }^{1}$, Xingkai Zhang ${ }^{1} \&$ Yindi Zhang ${ }^{1}$ \\ ${ }^{1}$ School of Petroleum Engineering, Yangtze University, Wuhan 430100, China; \\ ${ }^{2}$ School of Chemical Engineering and Technology, Xi'an Jiaotong University, Xi'an 710049, China \\ *Corresponding authors, E-mail: shibaoch@126.con; jjwei@mail.xjtu.edu.cn
}

As one of high energy consumption pumps, pump used for solid-liquid two-phase mixture transportation, such as slurry pump and mud pump, is extensively used in the hydraulic transportation of solid-liquid mixture through pipes in various fields. The erosion wear in slurry pumps has been identified as a critical issue during transportation of slurry as it affects the equipment performance and reduces its reliability and operation life, leading to the waste of energy. Many studies indicate that the performance and efficiency of solid-liquid two-phase centrifugal pump is controlled by the two-phase flow pattern inside the pump, so understanding flow characteristics and abrasion mechanism has important significance to improve the hydraulic design of the impeller for decreasing the abrasion and increasing the service life of the pump. In the present study, based on solid-liquid two-phase flow as object, an improved experimental facility without agitation was developed can improve the test precision by eliminating the influence of agitation on flow inside pump and improving particle-liquid two-phase flow circulation way. On the base of experimental studies, POD methods were applied to analysis the liquid turbulence modulation by solid-particles and the behavior of large eddy structures will be clearly understood. Thus, the theoretical system of the solid-liquid two-phase flow will be further improved. And a theoretical principle for the solid-liquid two-phase pump optimal design will be presented.

The following conclusions are obtained: ( $\mathrm{i}$ ) a superior PIV two-phase test platform than that in the previous literature is designed and it can be very good to realize a stable solid-liquid two-phase flow measurement of centrifugal pump. Compared with the traditional platform, the results obtained by the novel solid-liquid two-phase centrifugal pump PIV test platform has a very high accuracy and it has a good reference value to understand flow mechanism of a centrifugal slurry pump. The two particle images with and without agitating are shown in Figure 5. As can be seen that a large number of bubbles are brought into the circulation line (Figure 5(a)), which is caused by a higher stirring speed for suspending solid particles, and thus the actual flow images shows a gas-liquid-solid three-phase flow. However, for the present test facility without stirring, there is no such problem, and the original image is very clear without bubbles. (ii ) The POD analyses show that the solid-particles injection has an important influence on the large scale turbulence structures which contain a large amount of energy. The modal energy distribution as a function of the POD mode number is displayed in Figure 10. The energy contribution of these large scale structures to the total energy becomes larger due to the injection of solid-particles, whereas the contribution of the small scale structures, which denotes the energy dissipation, becomes smaller. This is why the low solid-phase volume concentration has higher pump head and efficiency. The addition of solid-particle increases the friction loss, but reduces the flow separation loss and inhibits the development of small scale vortex. However, with the increase of solid-particle concentration, the friction loss increases is greater than the flow separation loss reduction, and thus pump head and efficiency decrease. The above phenomena show fully that the solid-particles addition has an important influence on the large scale energetic eddy structures. This is confirmed by the measurement results as shown in Figure 11.

solid-liquid two-phase flow, PIV test, flow characteristics, turbulence modulation

doi: 10.1360/N972017-01073 